\title{
Optical Tamm States at the Interface between Photonic Crystal and Gyroid Layer
}

\author{
Rashid G. Bikbaev ${ }^{1, *}$, Stepan Ya. Vetrov ${ }^{1,2}$, and Ivan V. Timofeev ${ }^{2,3}$ \\ ${ }^{1}$ Institute of Engineering Physics and Radio Electronics, Siberian Federal University, Krasnoyarsk 660041, Russia \\ ${ }^{2}$ Kirensky Institute of Physics, Siberian Branch of the Russian Academy of Sciences, Krasnoyarsk 660036, Russia \\ ${ }^{3}$ Laboratory for Nonlinear Optics and Spectroscopy, Siberian Federal University, Krasnoyarsk 660041, Russia \\ *Corresponding author: Rashid-G-Bikbaev@ya.ru
}

Compiled August 22, 2017

\begin{abstract}
The spectral properties of a one-dimensional photonic crystal bounded by an absorbing plasmonic gyroid layer are investigated. The gyroid material is a foam-like metallic three-dimensionally periodic curved film with zero mean curvature at every point. We calculate the transmittance and reflectance spectra at the normal and oblique light incidence. The possibility of the Tamm state formation at the interface between a photonic crystal and a plasmonic gyroid layer caused by the negative real part of the gyroid permittivity is demonstrated for the first time. Specific features of field localization at the Tamm state frequencies are discussed. It is shown that the spectral and polarization properties of the optical Tamm states are highly sensitive to the change in the angle of incidence and in the refractive index of a medium filling the gyroid cavities. () 2017 Optical Society of America
\end{abstract}

OCIS codes: (160.5298) Photonic crystals; (160.3918) Metamaterials;(250.5403) Plasmonics.

http://dx.doi.org/10.1364/ao.XX.XXXXXX

\section{INTRODUCTION}

In recent years, there has been an increased interest in a special type of surface electromagnetic states called the optical Tamm states (OTSs) [1]. These states are analogous to the Tamm states in condensed matter physics. The OTS can be excited between two different photonic crystals (PCs) with the overlapping band gaps [2] or between a PC and a medium with negative permittivity $\epsilon[3,4]$. The surface electromagnetic wave at the interface between a PC and a conductor medium with $\epsilon<0$ is coupled with a surface plasmon, i.e., oscillations of free electrons near the conductor surface. The OTS can be experimentally observed as narrow peak in the transmittance spectrum $[5,6]$.

The OTSs are promising for application in sensors and optical switches [7], Faraday- and Kerr-effect amplifiers [8], organic solar cells [9], absorbers [10], thermal emitters [11] . Symonds et al. [12] experimentally demonstrated a laser based on the Tamm structure consisting of quantum wells embedded in a Bragg reflector with the silver-coated surface. Gazzano et al. [13] experimentally showed the possibility of implementation of a single-photon source on the basis of confined Tamm plasmon modes. In [6, 14-16], the OTSs in magnetophotonic crystals were investigated. Gessler et al. [17] studied the electrooptically tunable Tamm plasmon exciton polaritons. In our previous work [18], we examined the OTS formation at the interface between a PC bounded from its one or two sides by strongly anisotropic nanocomposite layers [19, 20]. In [21], we proposed a mechanism of the formation of two Tamm states at the interface between a superlattice and a nanoporous silver layer. In [22], the analog of the surface state in a structure containing a cholesteric liquid crystal was found. It was shown that the condition for its existence is the presence of a quarterwavelength layer between the cholesteric and metal due to the polarization properties of cholesterics atypical of scalar structures. Later, the chiral OTS was found [23] at the interface between a cholesteric and a metal-dielectric nanocomposite layer [24] acting as a polarization-preserving anisotropic mirror [25].

In addition, of great interest are the gyroid materials, i.e., the structures infinitely connecting and repeating in all the three dimensions and having the minimum possible surface (Fig. 1a). They were described first by Alan Schoen in 1970 [26]. The technique for fabricating such structures based on self-assembling multicomponent polymers was described in detail in [27-30]. Gyroids are formed from plasmon materials (silver and gold), as well as from titanium nitride TiN, which is chosen due to its low absorption in the visible spectral range [31]. Since the gyroid materials are periodic in three spatial dimensions, they are used to create three-dimensional PCs [32]. The gyroid materials occur in nature. Study of the parides sesostris butterfly [33] showed that its wing scales have the gyroid structure. Such natural periodic structures with a period of about $300 \mathrm{~nm}$ are called the biological PCs [34-36]. In this work, we investigate 
the properties of the OTSs localized at the edge of a PC bounded by a plasmon gyroid layer (PGL). The gyroid layer is a porous silver matrix filled with air or other pore filler. We study the possibility of controlling the OTS characteristics by varying the parameters of the gyroid layer.

\section{DESCRIPTION OF THE MODEL}

We consider the structure representing a layered medium conjugated with a finite plasmon gyroid layer (PGL) (Fig. 1). The PC unit cell is formed from materials $a$ and $b$ with the respective layer thicknesses and permittivities $d_{a}, \epsilon_{a}$ and $d_{b}, \epsilon_{b}$. The PC structure is surrounded by vacuum.
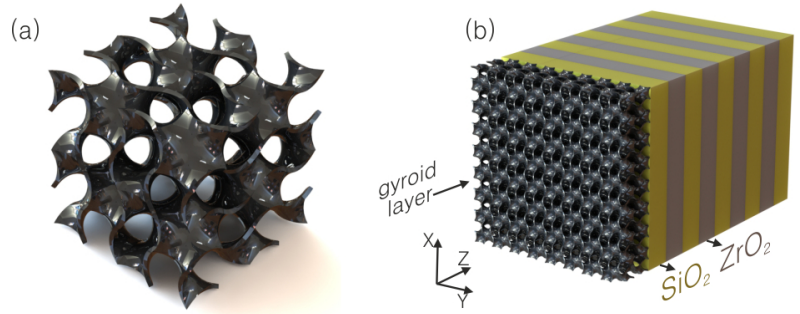

Fig. 1. Model of a plasmon gyroid material (a) and schematic of a 1D PC conjugated with the plasmon gyroid layer (b)

Maxwell's equations are reduced to Helmholtz equation. For $p$-polarized wave propagating in the $x z$ plane in a layered medium of isotropic materials stacked along the $z$-direction:

$$
\begin{array}{r}
{\left[\frac{d^{2}}{d z^{2}}-\left(k^{2}-\frac{\epsilon \omega^{2}}{c^{2}}\right)\right] E_{x}(z)=0,} \\
H_{y}(z)=\frac{i \omega}{c} \frac{\epsilon d E_{x} / d z}{c\left(k^{2}-\epsilon \omega^{2} / c^{2}\right)},
\end{array}
$$

where $\theta$ is the angle of incidence, $k=k_{x}=n(\omega / c) \sin (\theta)$ is the tangential wave number along the $x$-axis, $c$ is the speed of light and $\omega$ is the frequency.

The permittivity of the plasmon gyroid material with thickness $d_{g y r}$ is determined as [37]

$$
\epsilon_{g y r}(\omega)=\frac{l_{g} \sqrt{2}}{a}\left[1-\left(\frac{4 r_{g}}{\lambda_{g}}\right)^{2}\left(\frac{\pi \sqrt{-\epsilon_{m}(\omega)}}{2 \sqrt{2} n}-1\right)^{2}\right] .
$$

Expression (2) was obtained using tri-helical model for nanoplasmonic gyroid metamaterials that was proposed in [38]. In this model, the unit cell is changed for three plasmon nanohelices oriented in all the three spatial directions (Fig. 2a). In (2), the wire turn length is

$$
l_{g}=\sqrt{(2 \pi R)^{2}+a^{2}}=a \sqrt{\frac{\pi^{2}(\sqrt{2}-1)^{2}}{4}+1}
$$

where $a$ is the unit cell size,

$$
R=\frac{(\sqrt{2}-1)^{2}}{4}+1
$$

is the helix radius, and

$$
r_{g}=a \sqrt{f} \frac{\sqrt[4]{2}}{\pi\left[\sqrt{2+\pi^{2}}+\sqrt{2+(3+2 \sqrt{2}) \pi^{2}}\right]}
$$

is the helix wire radius ( $f$ is the metal volume fraction and $n$ is the refractive index of a medium filling the gyroid). The effective plasmon frequency of an ideal gyroid expressed through the unit cell size and volume fraction of a plasmon material is determined as

$$
\lambda_{g}=1.15 a \sqrt{1-0.65 \ln f} .
$$

We obtain the permittivity of a metal using the Drude approximation

$$
\epsilon_{m}(\omega)=\epsilon_{0}-\frac{\omega_{p}^{2}}{\omega(\omega+i \gamma)}
$$

where $\epsilon_{0}$ is the constant that takes into account the contributions of interband transitions of bound electrons, $\omega_{p}$ is the plasma frequency, and $\gamma$ is the reciprocal electron relaxation time. It should be noted that this model allows describing with the maximum accuracy the optical properties of plasmon gyroid structures, which was experimentally confirmed in $[37,39]$. (a)

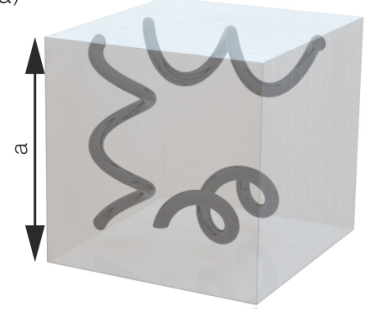

(b)

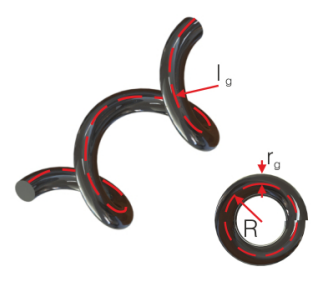

Fig. 2. Gyroid unit cell for the tri-helical model (a) and helix geometrical sizes (b)

The change in the light field upon its passing through each structural layer is determined by the second-order transfer matrix [40] and the transfer matrix of the entire structure, which relates the amplitudes of the incident and outgoing waves, is a product of the $2 \times 2$ matrices

$$
\hat{M}=T_{01} T_{12 \ldots} T_{N-1, N} T_{N, S}
$$

where the transfer matrix is

$$
T_{n-1, n}=\frac{1}{2}\left(\begin{array}{cc}
(1+h) e^{-i \alpha_{n} \gamma_{n}} & (1-h) e^{i \alpha_{n} \gamma_{n}} \\
(1-h) e^{-i \alpha_{n} \gamma_{n}} & (1+h) e^{i \alpha_{n} \gamma_{n}}
\end{array}\right),
$$

Here $\left.h=\sqrt{\varepsilon_{n} / \varepsilon_{n-1}}, \varepsilon(n)\right)$ is the permittivity of the $n$th layer, $\alpha_{n}=(\omega / c) \sqrt{\varepsilon(n)}, c$ is the speed of light, $\gamma_{n}=\mathrm{z}_{n}-\mathrm{z}_{n-1}$ are the layer thicknesses $(n=1,2, \ldots, N), z_{n}$ is the coordinate of the interface between the $n$th layer and the $(n+1)$ th layer adjacent from the right, and $\gamma_{N+1}=0$. The transfer matrix for the orthogonally polarized wave is obtained from (9) by replacing $h$ for $\sqrt{\varepsilon_{n-1} / \varepsilon_{n}}$. The energy transmittances, reflectances, and absorbances are determined as

$T(\omega)=\frac{1}{\left|\hat{M}_{11}\right|^{2}}, \quad R(\omega)=\frac{\left|\hat{M}_{21}\right|^{2}}{\left|\hat{M}_{11}\right|^{2}} . \quad A(\omega)=1-T(\omega)-R(\omega)$.

where $\hat{M}_{11}, \hat{M}_{21}$ - are the elements of matrix $\hat{M}$.

\section{RESULTS AND DISCUSSION}

First we investigate the OTS implemented in the form of a standing surface wave localized at the PC/PGL interface at normal incidence of light on structure. The alternating layer materials in the PC under study are silicon dioxide $\mathrm{SiO}_{2}$ with a permittivity 
of $\epsilon_{a}=2.10$ and zirconium dioxide $\mathrm{ZrO}_{2}$ with a permittivity of $\epsilon_{b}=4.16$. The respective layer thicknesses are $d_{a}=150 \mathrm{~nm}$ and $d_{b}=120 \mathrm{~nm}$ and the number of PC layers is $N=21$. The plasmon gyroid layer with a thickness of $d_{g y r}=133 \mathrm{~nm}$ consists of a silver matrix with a filler and has the complex permittivity $\epsilon_{g y r}(\omega)$

$$
\epsilon_{g y r}(\omega)=\Re \epsilon_{g y r}(\omega)+i \Im \epsilon_{g y r}(\omega) .
$$

The parameters of silver are $\epsilon_{0}(\omega)=5, \omega_{p}=9 \mathrm{eV}$, and $\gamma=0.02 \mathrm{eV}$. It should be noted that this gyroid material is the especially attractive nanocomposite, since its dispersion properties can be effectively controlled without changing the geometrical parameters and gyroid material. This is possible by changing the refractive index of a gyroid filler [39]. The optical properties of the gyroid were studied at a fixed unit cell size of $a=35$ $\mathrm{nm}$ and a plasmon material volume fraction of $f=30 \%$ (the values were taken from [37]). The frequency dependences of the real and imaginary parts of the permittivity calculated using formula (2) show that with increasing refractive index of a filler in the gyroid film the $\Im \epsilon_{g y r}(\omega)$ curve changes insignificantly. The $\Re \epsilon_{g y r}(\omega)$ curve is noticeably modified, and the frequency region narrows, where the inequality $\Re \epsilon_{g y r}(\omega)<0$ is valid. Fig. 3a shows the frequency dependences $\Re \epsilon_{g y r}(\omega)$ for silver and plasmon gyroid material at different refractive indices $n$ of the filler.
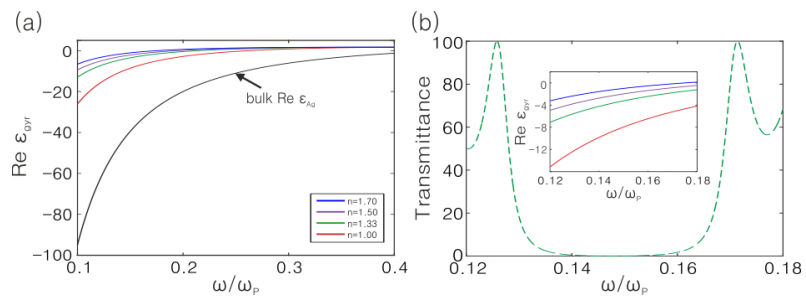

Fig. 3. Dependence of the real part $\Re \epsilon_{g y r}(\omega)$ of the effective permittivity $\epsilon_{g y r}(\omega)$ on normalized frequency $\omega / \omega_{p}$ at different refractive indices $\mathrm{n}$ of the filler (a) and transmittance spectrum of the initial PC (dashed line)(b). Inset: frequency dependence of the real part of the gyroid permittivity

It can be seen from Fig. $3 b$ that the variation in the refractive index of the filler is accompanied by a significant change in the permittivity in the frequency range corresponding to the PC band gap. It was established that at the specified parameters of the lattice and gyroid layer, the optical Tamm state at $n=1$ is not implemented. However, the localized state can be obtained in all the four cases by changing the thickness of the layer adjacent to the PGL. The calculation showed that the OTS is formed for every of the four refractive indices, e.g., at a first PC layer thickness of $d_{\text {first }}=200 \mathrm{~nm}$ (Fig. 4).

It can be seen from the figure that there are transmittance bands corresponding to the Tamm states localized at the PGL/PC interface near the high-frequency PC band gap edge. The obtained OTSs exist in the frequency range where the real part of the permittivity is negative. The OTS frequencies and effective permittivities are given in Table 1.

Now, let us consider the specific features of the transmittance and reflectance spectra of the PGL/PC system at different refractive indices of the filler for the case when the first PC layer thickness is invariable and equal to the initial thickness, i.e., 150 nm (Fig. 5).

It can be seen from Fig. 5a that the transmittance at the OTS frequency can be effectively controlled by changing the
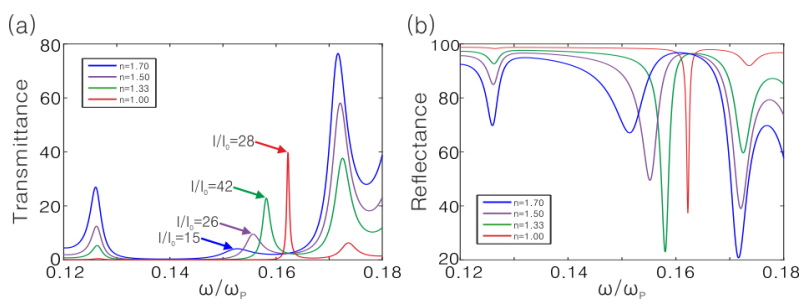

Fig. 4. Transmittance (a) and reflectance (b) spectra for the PC conjugated with the PGL at a first PC layer thickness of $d_{\text {first }}=200 \mathrm{~nm}$ and different refractive indices $n$ of the filler. The PGL thickness is $d_{g y r}=133 \mathrm{~nm}$ and the rest parameters are the same as in Fig. 3. The arrow captions in Fig. 4 show the intensities normalized to the input intensity in the transmittance curve maxima. The transmittance maxima correspond to the OTS frequencies

Table 1. OTS frequencies, wavelengths, and permittivities for the case illustrated in Fig. 4

\begin{tabular}{cccc}
\hline$n$ & $\omega / \omega_{p}$ & $\lambda(n m)$ & $\epsilon_{\text {gyr }}$ \\
\hline 1 & 0.1622 & 846.3 & $-6.64+0.16 i$ \\
1.33 & 0.1582 & 867.7 & $-2.77+0.09 i$ \\
1.5 & 0.1556 & 882.2 & $-1.76+0.07 i$ \\
1.7 & 0.1527 & 899 & $-0.94+0.06 i$ \\
\hline
\end{tabular}
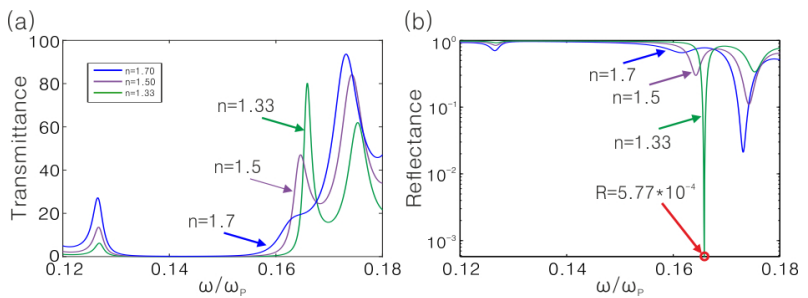

Fig. 5. Transmittance (a) and reflectance (b) spectra for the PC conjugated with the PGL at different refractive index $n$ of the filler. The PGL thickness is $d_{g y r}=133 \mathrm{~nm}$ and $d_{\text {first }}=150 \mathrm{~nm}$
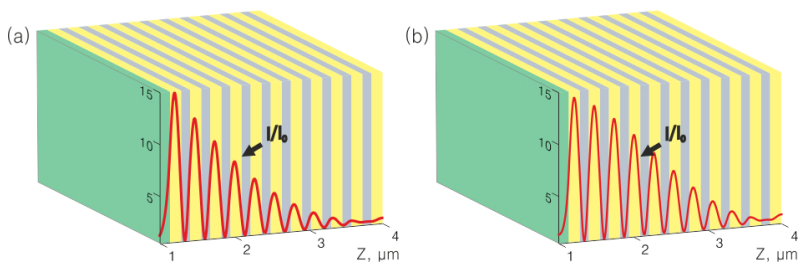

Fig. 6. Schematic of a 1D PC conjugated with the plasmon gyroid layer and the field intensity distribution at the OTS frequency. The PGL thickness is $d_{g y r}=133 \mathrm{~nm}$; (a) $n=1.5$ and (b) $n=1.33$. The rest parameters are the same as in Fig 5 
refractive index of the filler. In particular, the change of $n$ by $11.3 \%$ (from $n=1.33$ to $n=1.5$ ) leads to a decrease in the transmittance at the OTS frequency by a factor of 1.7 (from 80 to $47 \%$ ). With a further increase in the $n$ value, the OTS vanishes. This is caused by a strong shift of the dispersion curve $\Re \epsilon_{g y r}(\omega)$ to the low-frequency region and a decrease in the reflectance of the plasmon gyroid film. However, the reflectance spectra have a unique feature. It can be seen from Fig. $5 b$ that the reflectance at the OTS frequency changes by a factor of 600 (from 0.05 to $30 \%$ ). The reflectance is more sensitive to the refractive index variation than the transmittance. The low reflectance at $n=1.33$ is caused by the high absorption (23.5\%) of the incident radiation at the OTS frequency. The spatial distribution of the electric field intensity at the OTS frequencies corresponding to $n=1.5$ and $n=1.33$ are presented in Fig. 6 .

It can be seen that field localization at the OTS frequencies is nearly the same. In both cases, the light field in the OTS is localized in the region commensurable with the wavelength. One of the effective ways of controlling the energy spectra is changing the radiation incidence angle (Fig. 7).

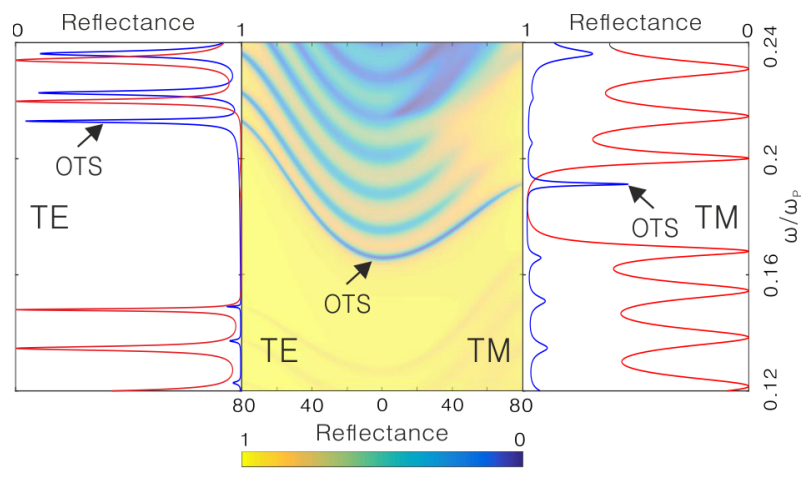

Fig. 7. Angular and frequency dependences of the reflectance spectra of the PGL/PC system for the TE and TM modes (in the middle) and reflectance spectra of the PGL/PC layer at an angle of incidence of $80 \mathrm{deg}$. for the TE (on the left) and TM (on the right) modes. The red line shows the initial PC spectra and the blue line, the spectra of the PC conjugated with the gyroid layer. The gyroid layer thickness is $d_{g y r}=133 \mathrm{~nm}$ and $n=1.33$

Study of the reflectance spectra showed that for the TE modes the change in the angle of incidence leads to the broadening of the PC band gap and significant shift of the OTS to the shortwavelength region. For the TM modes, the picture is analogous, except for the fact that the band gap does not increase, but decreases. In addition, note that at an angle of incidence of 80 deg, the OTS shift to the short-wavelength region was $184.3 \mathrm{~nm}$ for the TM modes and $109.8 \mathrm{~nm}$ for the TE modes (Fig. 7). Such a strong polarization sensitivity of the structure allows effective controlling the spectral characteristics of the OTSs and using them in optical sensors.

\section{CONCLUSION}

Thus, using the transfer matrix technique, we calculated and analyzed for the first time the transmittance and reflectance spectra of a PC conjugated with the plasmon gyroid layer. The initial gyroid layer is a porous silver matrix filled with air. The possibility of implementing the OTSs at the PGL/PC interface was demonstrated. It was established that the transmittance and reflectance at the OTS frequencies of the PGL/PC system are highly sensitive to the variation in the refractive index of the gyroid filler. In particular, the change of $n$ by $11.3 \%$ (from $n=1.33$ to $n=1.5$ ) leads to an increase in the reflection at the OTS frequency by a factor of 600 (Fig. 5b). In addition, the high polarization and angular sensitivity of the reflectance spectra at the OTS frequencies was demonstrated (Fig. 7). Thus, the characteristics of the OTSs can be effectively controlled, which allows using these states in optical sensors and polarizers.

This work was financially sponsored by Russian Foundation for Basic Research, Government of Krasnoyarsk Territory, Krasnoyarsk Region Science and Technology Support Fund to the research project № 17-42-240464. R.G.B. acknowledges financial support from Russian Foundation for Basic Research, Government of Krasnoyarsk Territory, Krasnoyarsk Region Science and Technology Support Fund to the research project № 16-42-243065.

\section{REFERENCES}

1. A. P. Vinogradov, A. V. Dorofeenko, A. M. Merzlikin, and A. a. Lisyansky, "Surface states in photonic crystals," Physics-Uspekhi 53, 243-256 (2010).

2. A. V. Kavokin, I. A. Shelykh, and G. Malpuech, "Lossless interface modes at the boundary between two periodic dielectric structures," Physical Review B 72, 233102 (2005).

3. M. Kaliteevski, I. Iorsh, S. Brand, R. A. Abram, J. M. Chamberlain, A. V. Kavokin, and I. A. Shelykh, "Tamm plasmon-polaritons: Possible electromagnetic states at the interface of a metal and a dielectric Bragg mirror," Physical Review B 76, 165415 (2007).

4. S. Y. Vetrov, R. G. Bikbaev, and I. V. Timofeev, "Optical Tamm states at the interface between a photonic crystal and a nanocomposite with resonance dispersion," Journal of Experimental and Theoretical Physics 117, 988-998 (2013)

5. M. E. Sasin, R. P. Seisyan, M. A. Kalitteevski, S. Brand, R. A. Abram, J. M. Chamberlain, A. Y. Egorov, A. P. Vasil'ev, V. S. Mikhrin, and A. V. Kavokin, "Tamm plasmon polaritons: Slow and spatially compact light," Applied Physics Letters 92, 251112 (2008).

6. T. Goto, A. V. Dorofeenko, A. M. Merzlikin, A. V. Baryshev, A. P. Vinogradov, M. Inoue, A. A. Lisyansky, and A. B. Granovsky, "Optical tamm states in one-dimensional magnetophotonic structures," Physical Review Letters 101, 14-16 (2008).

7. W. Zhang and S. Yu, "Bistable switching using an optical Tamm cavity with a Kerr medium," Optics Communications 283, 2622-2626 (2010).

8. A. P. Vinogradov, A. V. Dorofeenko, S. G. Erokhin, M. Inoue, A. A. Lisyansky, A. M. Merzlikin, and A. B. Granovsky, "Surface state peculiarities in one-dimensional photonic crystal interfaces," Physical Review B 74, 045128 (2006).

9. X.-L. Zhang, J.-F. Song, X.-B. Li, J. Feng, and H.-B. Sun, "Optical Tamm states enhanced broad-band absorption of organic solar cells," Applied Physics Letters 101, 243901 (2012).

10. Y. Gong, X. Liu, L. Wang, H. Lu, and G. Wang, "Multiple responses of TPP-assisted near-perfect absorption in metal/Fibonacci quasiperiodic photonic crystal," Optics Express 19, 9759 (2011).

11. Z.-Y. Yang, S. Ishii, T. Yokoyama, T. D. Dao, M.-G. Sun, T. Nagao, and K.-P. Chen, "Tamm plasmon selective thermal emitters," Optics Letters 41, 4453 (2016).

12. C. Symonds, G. Lheureux, J. P. Hugonin, J. J. Greffet, J. Laverdant, G. Brucoli, A. Lemaitre, P. Senellart, and J. Bellessa, "Confined Tamm Plasmon Lasers," Nano Letters 13, 3179-3184 (2013).

13. O. Gazzano, S. Michaelis de Vasconcellos, K. Gauthron, C. Symonds, P. Voisin, J. Bellessa, A. Lemaître, and P. Senellart, "Single photon source using confined Tamm plasmon modes," Applied Physics Letters 100, 232111 (2012).

14. H. Y. Dong, J. Wang, and T. J. Cui, "One-way Tamm plasmon polaritons at the interface between magnetophotonic crystals and conducting metal oxides," Physical Review B 87, 045406 (2013). 
15. H. Da, Q. Bao, R. Sanaei, J. Teng, K. P. Loh, F. J. Garcia-Vidal, and C.-W. Qiu, "Monolayer graphene photonic metastructures: Giant Faraday rotation and nearly perfect transmission," Physical Review B 88, 205405 (2013).

16. N. E. Khokhlov, A. R. Prokopov, A. N. Shaposhnikov, V. N. Berzhansky, M. A. Kozhaev, S. N. Andreev, A. P. Ravishankar, V. G. Achanta, D. A. Bykov, A. K. Zvezdin, and V. I. Belotelov, "Photonic crystals with plasmonic patterns: novel type of the heterostructures for enhanced magneto-optical activity," Journal of Physics D: Applied Physics 48, 095001 (2015).

17. J. Gessler, V. Baumann, M. Emmerling, M. Amthor, K. Winkler, S. Höfling, C. Schneider, and M. Kamp, "Electro optical tuning of Tamm-plasmon exciton-polaritons," Applied Physics Letters 105, 181107 (2014).

18. S. Y. Vetrov, R. G. Bikbaev, and I. V. Timofeev, "The optical Tamm states at the edges of a photonic crystal bounded by one or two layers of a strongly anisotropic nanocomposite," Optics Communications 395, 275-281 (2017).

19. Y. Dadoenkova, I. Glukhov, S. Moiseev, V. Svetukhin, A. Zhukov, and I. Zolotovskii, "Optical generation in an amplifying photonic crystal with an embedded nanocomposite polarizer," Optics Communications 389, 1-4 (2017).

20. S. G. Moiseev, V. A. Ostatochnikov, and D. I. Sementsov, "Defect mode suppression in a photonic crystal structure with a resonance nanocomposite layer," Quantum Electronics 42, 557-560 (2012).

21. R. G. Bikbaev, S. Y. Vetrov, and I. V. Timofeev, "The optical Tamm states at the interface between a photonic crystal and nanoporous silver," Journal of Optics 19, 015104 (2017).

22. S. Y. Vetrov, M. V. Pyatnov, and I. V. Timofeev, "Surface modes in "photonic cholesteric liquid crystal-phase plate-metal" structure," Optics Letters 39, 2743 (2014).

23. I. V. Timofeev and S. Y. Vetrov, "Chiral optical Tamm states at the boundary of the medium with helical symmetry of the dielectric tensor," JETP Letters 104, 380-383 (2016).

24. S. G. Moiseev and V. A. Ostatochnikov, "Defect modes of onedimensional photonic-crystal structure with a resonance nanocomposite layer," Quantum Electronics 46, 743-748 (2016).

25. N. V. Rudakova, I. V. Timofeev, P. S. Pankin, and S. Y. Vetrov, "Polarization-preserving anisotropic mirror on the basis of metal-dielectric nanocomposite," Bulletin of the Russian Academy of Sciences: Physics 81, 5-9 (2017).

26. A. H. Schoen, "Infinite periodic minimal surfaces without selfintersections," Nasa Technical Note D-5541 p. 92 (1970).

27. S. Vignolini, N. A. Yufa, P. S. Cunha, S. Guldin, I. Rushkin, M. Stefik, K. Hur, U. Wiesner, J. J. Baumberg, and U. Steiner, "A 3D Optical Metamaterial Made by Self-Assembly," Advanced Materials 24, OP23OP27 (2012).

28. I. Vukovic, G. T. Brinke, and K. Loos, "Block copolymer templatedirected synthesis of well-ordered metallic nanostructures," Polymer 54, 2591-2605 (2013).

29. H.-Y. Hsueh, H.-Y. Chen, Y.-C. Ling, W.-S. Huang, Y.-C. Hung, S. Gwo, and R.-M. Ho, "A polymer-based SERS-active substrate with gyroidstructured gold multibranches," J. Mater. Chem. C 2, 4667-4675 (2014).

30. Y. Li, B. P. Bastakoti, and Y. Yamauchi, "Research Update: Triblock copolymers as templates to synthesize inorganic nanoporous materials," APL Materials 4, 040703 (2016).

31. S. Prayakarao, S. Robbins, N. Kinsey, A. Boltasseva, V. M. Shalaev, U. B. Wiesner, C. E. Bonner, R. Hussain, N. Noginova, and M. A. Noginov, "Gyroidal titanium nitride as nonmetallic metamaterial," Optical Materials Express 5, 1316 (2015).

32. K. Hur, Y. Francescato, V. Giannini, S. A. Maier, R. G. Hennig, and U. Wiesner, "Three-Dimensionally Isotropic Negative Refractive Index Materials from Block Copolymer Self-Assembled Chiral Gyroid Networks," Angewandte Chemie 123, 12191-12195 (2011).

33. B. D. Wilts, K. Michielsen, H. De Raedt, and D. G. Stavenga, "Iridescence and spectral filtering of the gyroid-type photonic crystals in Parides sesostris wing scales," Interface Focus 2, 681-687 (2012).

34. K. Michielsen and D. Stavenga, "Gyroid cuticular structures in butterfly wing scales: biological photonic crystals," Journal of The Royal Society Interface 5, 85-94 (2008).

35. V. Saranathan, C. O. Osuji, S. G. J. Mochrie, H. Noh, and S. Narayanan, "Crystals in Butterfly Wing Scales," Proceedings of the National Academy of Sciences of the United States of America 107, 11676-11681 (2010).

36. L. Poladian, S. Wickham, K. Lee, and M. C. Large, "Iridescence from photonic crystals and its suppression in butterfly scales," Journal of The Royal Society Interface 6, S233-S242 (2009).

37. P. Farah, A. Demetriadou, S. Salvatore, S. Vignolini, M. Stefik, U. Wiesner, O. Hess, U. Steiner, V. K. Valev, and J. J. Baumberg, "Ultrafast Nonlinear Response of Gold Gyroid Three-Dimensional Metamaterials," Physical Review Applied 2, 044002 (2014).

38. A. Demetriadou, S. S. Oh, S. Wuestner, and O. Hess, "A tri-helical model for nanoplasmonic gyroid metamaterials," New Journal of Physics 14, 083032 (2012).

39. S. Salvatore, A. Demetriadou, S. Vignolini, S. S. Oh, S. Wuestner, N. A. Yufa, M. Stefik, U. Wiesner, J. J. Baumberg, O. Hess, and U. Steiner, "Tunable 3D Extended Self-Assembled Gold Metamaterials with Enhanced Light Transmission," Advanced Materials 25, 2713-2716 (2013).

40. P. Yeh, "Electromagnetic propagation in birefringent layered media," Journal of the Optical Society of America 69, 742 (1979). 